\title{
A CASE OF ATYPICAL MULTIPLE SCLEROSIS WITH
}

BULBAR PARALYSIS ${ }^{1}$

\section{By Sigmund Krumholz, M.D.}

This girl is eighteen years old, born in Chicago, doing general housework at home.

Mother and two older sisters and herself are afflicted with otosclerosis. Two other children died, one at nineteen years of cardiac rheumatism, the other at thirteen months in a convulsive attack; otherwise family history negative.

Birth and early childhood of the patient normal. At three years had measles and diphtheria; at age of six years was operated on for suppurative cervical adenitis on the left side of the neck. At twelve years had tonsillectomy performed, and again in March, I9r3, had undergone another tonsil and adenoid operation. Since the age of thirteen years has had a spastic torticollis. Her hereditary otosclerosis gradually developed, beginning at the age of six years. Intelligence normal. Menses regular. Habits good.

About July, I912, suffered a slight contusion of the left side of neck, and dates the onset of present complaint to this accident. The patient complained of a dull continuous pain on the left side of the back of the neck, radiating upwards and homolaterally, and for the last three months (about one year after date of onset) the pain was also present at the right side of the neck.

In May, I913, the patient experienced a neuralgic pain on left side of the forehead, accompanied by hoarseness and dry cough, which was soon followed by impairment of speech and voice. The latter two functions became gradually more affected, and about one month later the patient experienced difficulty in swallowing, so that liquids would at times partly regurgitate through the nose, and sometimes swallowing of food would excite a coughing spell. Fever was at no time observed.

On September 9, I9r3, Dr. Krumholz first saw the patient, at the request of Dr. Joseph C. Beck, and obtained the history just

1 Read before the Chicago Neurological Society, Dec. 17, 1914. 
described, and on examination found a bright, well-nourished and normally developed young girl. The history was gotten with some difficulty, on account of the combined deafness and dysarthria of the patient. Her speech was thick, indistinct and nasal in character, and her voice dysphonic. Left half of the tongue felt spongy, and was thinner than the right, presenting slight corrugations and marked fibrillary twitchings, and on protrusion it deviated to the left side. The velum palati hung down lower on the left than on the right side, and on phonation was not elevated. The uvula was drawn over towards the right side. Laryngoscopic examination by Dr. Joseph C. Beck discovered a paralysis of the left vocal cord. The patient found difficulty in the articulation of words which contained the linguals $r, 1$, n, etc., and pronounced them indistinctly, but the enunciation of the labials, p, b, w, etc., was not in any degree defective. On drinking water, there was some regurgitation through the nose. The sensation of the left side of the pharynx was not as acute as on the right. The eyeballs moved in all directions normally and without any nystagmus. The pupils were round, equal in size, and reacted readily and efficiently to light and convergence; no anophthalmos; no exophthalmos; the corneal and conjunctival reflexes were present. The eye grounds were normal. The upper and lower facial muscles contracted sufficiently and equally on both sides. The masseters well innervated. No disturbance to touch, pain and temperature sense on either side of the face and forehead.

The neck is short and thick like her mother's. The head is slightly drawn to the right. The face and chin directed slightly to the left and upward, but only when not self-conscious. The posterior muscles of the neck on the left side feel on paipation like one hard mass. Upon bending the head to the right, the left sterno-mastoid muscle becomes extremely tense. Rotation and side-to-side bending of the head is limited. The upper third of the left trapezius is slightly thinner and possibly weaker than the right. No fibrillary twitchings. There was some tenderness on pressure over the posterior part of the neck, especially on the left side, but no objective sensory disturbance to be detected over the neck and head, nor on any other part of the body.

At the root of the left side of the neck above the clavicle a slightly enlarged gland could be palpated, but no other glands. The lower end of the left sterno-mastoid muscle was markedly thickened; the thyroid was not enlarged. 
The reflexes of both patellar and Achilles tendons were exaggerated on both sides, more on the right side. No ankle clonus. Positive Babinski was only at times obtainable on the right, not on the left, side. Biceps, triceps, and periosteal reflexes were increased bilaterally, but a little livelier on the right side. The abdominal reflex was present.

September I3, I9I3, the physical findings and subjective symptoms remained unchanged. No distinct change in the taste sense. Temperature, $98.4^{\circ}$; pulse, 88 . The contractions of the neck muscles to the galvanic current were lightning-like in character on both sides. Heart, lungs, and abdominal viscera negative. No palpitation or dyspnea. There is a scar over the left antero-lateral side of the neck, due to the old operation for cervical adenitis.

September I5, 1913: Tuberculin test with Koch's O. T., gave a slight local reaction, but no constitutional effect was observed.

September 25, 1913: The sero-biological findings were: Wassermann in both blood serum and spinal fluid negative. The cell content and the globulin not in excess. Lange's colloidal gold test negative. No bacteria in spinal fluid.

Blood count: Lymphocytes, 40; large mononuclears, I3.3; large polynuclears, 33.3 ; eosinophiles, I3.3. Urinalysis negative.

November 14, I9I4: Blood finding same, except tubercular fixation faintly positive.

September 27, I9I3: The condition of patient considerably improved. Absence of dysphagia and regurgitation. The speech was much more distinct than previously, and the fibrillary twitchings of the tongue markedly diminished, and its consistency on the left side harder than on previous examination. The reflexes are about the same. No Babinski, but positive Gordon on right side. On examination of the eyes, a horizontal nystagmus could be seen distinctly on directing the patient to look to the side. This symptom could not be obtained on previous examinations.

Dr. Krumholz did not see the patient again for over one year, until November I4, I9 I4. Dr. J. C. Beck told him that he had operated on the patient November, 1913, which operation will be described by Dr. Pollock; and his notes, which Dr. Beck kindly furnished, give record that the patient feels better and that the pain in the back of the neck diminished considerably, but that the described paralysis of the tongue, soft palate and larynx did not change materially, that a haziness in the outline of the discs was 
to be noticed. In Dr. Krumholz's repeated examinations of the patient, November, I9I4, he found the unilateral glosso-laryngopalatine paralysis about the same as in September, I9I3, except that the tongue was more corrugated, and the optic discs were hazy in outline, and that the remote torticollis remained stationary.

In considering the differential diagnosis, cerebrospinal syphilis has to be excluded, on account of the absence of the general clinical luetic symptoms, and negative sero-biological report, which, to a great extent, also spoke against a pachymeningitis in the area of the exits of the affected nerves, or in the cervical region of the cord.

Aneurysm at the base or acute bulbar paralysis, due to hemorrhage, etc., had to be ruled out, since the patient had no cardiac disease, nor any etiologic factor producing arteritis.

Again, the absence of acute symptoms excluded polyencephalitis.

Chronic progressive bulbar paralysis had to be eliminated from the diagnosis on account of the remission of the symptoms, the youth of the patient, the limitation of the lesion to one side, and the absence of involvement of the facial.

Again, paralysis of the trunks of the pneumogastric and hypoglossal nerves caused by tumor pressure (for instance, enlarged tuberculous glands) from without at their exits, or along their course at the upper part of the neck, before their divergence from each other, had to be excluded, because the general picture of the disease did not conform with such diagnosis. In lesions at the jugular foramen (exit for ninth, tenth, and eleventh nerves) or in affections of their branches in their side-by-side course at the upper part of the neck, the laryngo-palatal paralysis is always accompanied by paralysis of the neck muscles (trapezius and sterno-cleido-mastoid). This patient has a remote acquired reflex spastic torticollis since her thirteenth year, probably due to muscular irritation, produced by cervical lymph nodes, but a recent paralysis of the spinal portion of the spinal accessory nerve could not be detected. In paralysis of this branch of the eleventh the scapula assumes a swinging position. The head is drawn towards the unaffected side, on account of the unopposed action of the healthy sterno-cleido-mastoid muscle, which is exclusively supplied by the eleventh nerve. In this case, as above stated, there is a limited side-to-side motion, and tension on the 
left sterno-mastoid and contraction of the trapezius, due to a spasm, but no flaring-out of the scapula, nor distinct bending of the head towards the healthy side. On electrical test, these muscles do not respond to the reaction of degeneration.

Again, polyneuritis of the branches of the tenth, eleventh and twelfth nerves had to be ruled out, because the general affections (influenza, diphtheria, etc.) which lead to lesions of these nerves usually produce bilateral paralysis; while tuberculous peripheral neuritis is very rare, and probably always secondary to tuberculous meningitis. This patient, with the exception of pain in the back of the neck, presented no symptoms of meningeal irritation.

Besides, the fibrillary twitching of the atrophic tongue, although occurring in neuritis, is usually a sign of nuclear affection.

Again, the exaggerated reflexes and transitory Babinski imply affection of the upper motor neuron. No extracranial lesion, with the exception of a vertebral compression of the cord, could have produced the endogenic neuron affection in our patient. The Roentgen pictures showed no lesion of the vertebræ and sku1l.

Again, the nystagmus cannot be explained upon a disease affecting the tenth, eleventh and twelfth nerves after their escape from the skull. This symptom, if not normal, represents a disturbed function of the vestibular nerve, or in the connection of its nucleus with the cerebellum. If we stretch our imagination and overlook the character of the nystagmus, then the otosclerosis could be held responsible for its presence. But in otosclerosis the involvement of the labyrinth is rare, and the character of the labyrinthine nystagmus is such that the slow component of the nystagmus is directed toward the side of the irritating lesion. In this patient the slow phase of the nystagmus is directed toward the median line, which, according to J. Gordon Wilson, is characteristic of an intracranial nystagmus.

The above enumerated diseases practically exhaust all that may be considered in differential diagnosis, and leave as a substratum of the disease in question some affection in the depth of the stem destroying the left nucleus ambiguus, the left nucleus hypoglossus, the left and possibly the right pyramidal tracts, and the vestibular nuclei. These anatomical structures are separated from each other by the interposition of important fiber systems (sensory fibers of the fifth nerve, fibers of pain and temperature 
sense to the body), the destruction of which would present characteristic objective sensory disturbance. These facts force the assumption that two or more lesions are responsible for the clinical syndrome of this disease. The behavior of the symptoms is characteristic of one of those diseases, the lesions of which are disseminated, namely, disseminated cerebrospinal syphilis, which was already excluded, and multiple sclerosis.

After the laryngo-glosso-palatine paralysis had distressed the girl for several weeks, it receded to a marked extent. At this examination, when the improvement was noticeable, Dr. Krumholz observed for the first time a distinct nystagmus. In October, I9I 3 , the outline of the optic discs appeared hazy and haziness is still present. This remission, intermission and appearance of new symptoms is most characteristic of multiple sclerosis. Atrophy and long intermissions are rare, but do occur. Oppenheim mentions in his text-book his observations of "hemiatrophia lingualis" in multiple sclerosis. Fuerstner published a case of multiple sclerosis with fibrillary tremor of the atrophic tongue, and on necropsy found a sclerotic process distributed in the medulla and hemispheres. Goodhart reports a case of multiple sclerosis in a twentyfour-year-old girl, who had sensory paresis in the hands and flaccid motor paralysis of the lower extremities, which gradually disappeared within six months, and after an intermission of seven years developed the classical symptom-complex of the disease. Kennedy reports two cases of multiple sclerosis with nuclear facial paralysis. My patient, No. 2 on the program, who, to my regret, failed to come here to-night, is almost a counterpart of Goodhart's case.

The finding of tuberculous glands at the operation is interesting, in which connection may be noted Stan Fleshen's recent preliminary report of eighteen cases of multiple sclerosis with distant tuberculous lesions, wherein he expressed the opinion that tuberculotis lesions in distant organs are probably the specific etiological factors of the disease. 